\title{
PARTICLE TRANSPORT PROCESSES
}

\author{
Georges Michaud \\ Departement de Physique \\ Université de Montréal \\ C. R. Proffitt \\ Space Telescope Science Institute
}

\begin{abstract}
The effect of gravitational settling and radiation driven diffusion on the evolution of stars near the main sequence is reviewed. New simplified formulae for calculating diffusion are proposed that improve on previous such formulae. The reliability of available diffusion coefficients is discussed and areas where further work is needed are identified. Newly available opacity calculations are used to estimate the effects of radiative acceleration on $\mathrm{Fe}$.

The size of the modifications to the evolution are shown to be modest: a reduction of order $10 \%$ on the evolutionary age of globular clusters. There are indications from the $\mathrm{Li}$ abundance in the high $\mathrm{T}_{\text {eff }}$ halo stars that some turbulence is present below the convection zone. Models calibrated using solar properties reproduce the $\mathrm{T}_{\text {eff }}$ at which the $\mathrm{Li}$ gap is observed in $\mathrm{F}$ stars as well as its depth without any arbitrary parameter. Similarly the presence of AmFm stars is explained over the $T_{\text {eff }}$ range where they are observed.
\end{abstract}

\section{ASTROPHYSICAL CONTEXT}

While it is a fundamental physical process, atomic diffusion was put aside in stellar evolution following Eddington's (1926) argumentation that the absence of extreme heavy element abundances ruled out the equilibrium configurations to which diffusion would lead in stellar interiors. He suggested that meridional circulation was a competing process and probably ruled out the effects of atomic diffusion.

It is only in the 1960 s that the role of atomic diffusion, driven by gravity (Aller and Chapman 1960) and differential radiation pressure (Michaud 1970), was reintroduced in main sequence stellar envelopes taking into consideration that the diffusion time scale is much shorter in external than in internal regions (Michaud et al. 1976). Since diffusion time scales are shorter than evolutionary time scales only in external stellar regions, anomalies should appear most strongly only in those stars where all competing hydrodynamical processes are reduced in external regions such as Ap stars. Equilibrium configurations for individual chemical species are never approached in stellar interiors.

It has since been shown that meridional circulation could compete with diffusion as Eddington suggested. Indeed, while the AmFm and the $\mathrm{HgMn}$ phenomena are observed up to equatorial rotation velocities of $V_{e} \sim 100 \mathrm{~km} \mathrm{~s}^{-1}$, a simple parameter free model taking diffusion and meridional circulation into account leads to an expected cut off velocity of $V_{c} \sim 100 \mathrm{~km} \mathrm{~s}^{-1}$ for the AmFm and $\mathrm{HgMn}$ phenomena (Michaud 1982, Charbonneau and Michaud 1988, 1991). 


\section{II- DIFFUSION EQUATIONS}

\section{Basic Formulae}

The most rigorous derivations of the equations for atomic diffusion start from the Boltzmann equation for the velocity distribution functions for each species of particle. A variety of techniques have been proposed to obtain approximate solutions to the Boltzmann equation (see, for instance, Chapman and Cowling 1970 and Burgers 1969). Usually the deviations from the ideal Maxwell-Boltzmann velocity distribution appropriate for statistical equilibrium in a uniform gas are small, but these deviations are essential for calculating higher order effects such as thermal diffusion.

There are many ways of expressing the resulting flow equations. It is often useful to treat two or three ions at a time and have a single diffusion velocity to solve as in Richer, Michaud and Proffitt (1992). For the general case of a multicomponent plasma, the expressions given by Burgers (1969) are convenient and we will use them for our discussion here. For each ionic species there is a force equation (eq. 18.1 of Burgers 1969),

$$
\frac{d P_{i}}{d r}+m_{i} n_{i} g+n_{i} z_{i}(e E)=\sum_{j} k_{i j}\left(v_{j}-v_{i}\right)+\sum K_{i j} z_{i j} \frac{m_{j} r_{i}-m_{i} r_{i}}{m_{i}+m_{j}} .
$$

A similar equation could be written for electrons or it may be deduced from the hydrostatic equation. For each species including the electrons there is a heat equation (eq. 18.2 of Burgers). To these is added the mass conservation equation (eq. 2.20 of Burgers) requiring that mass does not move with respect to the centre of mass. A similar equation may be written for the charge since only extremely small charge separations are allowed to develop. For $s$ different ionic species, this gives $2 s+3$ equations. The unknown quantities to be solved for in this system of equations are the velocities $\mathrm{v}$ and heat flux vectors $r$, for both the ions and electrons, and the value for the electric field.

The quantities $\mathrm{K}_{\mathrm{ij}}$ in the above equations are the resistance coefficients, and represent the effects of collisions between particles of types $i$ and $j$. They can be written as,

$$
k_{i j}=\frac{16}{3} n_{i} n_{j}\left(\frac{m_{i} m_{i}}{m_{i}+m_{j}}, \Omega_{i j}^{(11)}\right. \text {. }
$$

The heat flux terms involve additional collision integrals:

$$
z_{i j}=1-\frac{2 \Omega_{i j}^{(12)}}{5 \Omega_{i j}^{(11)}}, \quad z_{i j}^{\prime}=\frac{5}{2}-\frac{10 \Omega_{i j}^{(12)}-2 \Omega_{i j}^{(13)}}{5 \Omega_{i j}^{(11)}}
$$

and a similar one for $z_{i j}{ }^{*}$ (see eq. $11.7 \mathrm{c}$ of Burgers). The collision integrals $\Omega_{\mathrm{ij}}$ are discussed in the following section.

The $\mathrm{K}_{i j}$ 's are simply related to Chapman and Cowling's (1970) first approximation for diffusion coefficients. It has been shown (Burgers 1969, and Roussel-Dupre 1981) that, when Chapman and Cowling's second approximation to the diffusion coefficient and thermal diffusion terms are included, the method of Chapman is equivalent to that of Burgers. 


\section{Radiative Accelerations}

In the presence of a radiation flux, $g$ should be replaced by $g-g_{R}$ in equation (1). The competition between radiative acceleration, $g_{R}$, and gravity determines whether diffusion leads to over- or underabundances. Accurate radiative accelerations have only been calculated for a few elements. General formulae are available (Michaud et al. 1976; Alecian and Artru 1990) but are accurate to at most a factor of 3 (see Michaud 1987 for a discussion). Calculations are needed using more accurate data banks. The required atomic data is becoming available from the Opacity project and from the large compilations of Kurucz (1991). Detailed calculations are under way for $\mathrm{C}, \mathrm{N}, \mathrm{O}$.

For Fe, Alecian, Michaud and Tully (1992) have calculated radiative accelerations in detail in main sequence models with $T_{\text {eff }}=6700,8000$ and 10000 $\mathrm{K}$ and $\log \mathrm{g}=4.0$ and 4.3 and $\mathrm{a}$ number of $\mathrm{Fe}$ abundances. The atomic data available from the Opacity Project for Fe IX to Fe XVII allows calculating the accelerations over an important fraction of the envelope. In stars of $T_{\text {eff }}=6700 \mathrm{~K}$, radiative acceleration and gravity are nearly equal below the convection zone and only small $\mathrm{Fe}$ anomalies are expected. In cooler stars, underabundances of $\mathrm{Fe}$ are expected to materialize since gravity is always larger than $g_{R}$. They are expected to be small in so far as the progressive desaturation of the lines will lead to an increase of $g_{R}$. In stars of 8000 and $10000 \mathrm{~K}, g_{R}(\mathrm{Fe})$ is significantly larger than gravity over part of the envelope. If the envelope is stable enough, Fe overabundances will then appear over part of the envelope. Detailed evolutionary calculations are needed to determine where these overabundances appear. The Fe abundance is expected to modify the structure of the model since the recent results of Rogers and Iglesias (1992) show that over part of the envelope, iron is the main source of opacity.

\section{The Collision Integrals}

These integrals can be calculated by using the binary collision approximation, or the Fokker-Planck approximation, in which it is assumed that most collisions are sufficiently weak that the effects of two particle collisions can be summed, even if many particles are interacting simultaneously. When diffusion velocities are small compared to thermal velocities both approximations give the same result for sufficiently dilute plasmas. form

In general, for interactions between two particles, collision integrals of the

$\Omega_{i j}^{(\alpha \beta)}=\int_{0}^{\infty} \int_{0}^{\infty} e^{-g^{2}} g^{2 \beta+3}\left(1-\cos ^{\alpha} x_{i j}\right) b d b d g$

occur, with

$x_{i j}=\pi-2 \int_{r_{\min }}^{\infty} b d r\left\{r^{2}\left[1-\frac{b^{2}}{r^{2}}-\frac{v_{i j}(r)}{g^{2} k T}\right\}^{1 / 2}\right\}^{-1}$.

where $\mathrm{g}^{2}$ is the kinetic energy in the centre of mass of the colliding particles in units of $\mathrm{kT}$. For the Coulomb potential the above integrals become infinite due to the long range of the Coulomb force. In a real plasma the charged particles tend to polarize the surrounding medium, and the long range Coulomb interaction is cut off by this screening. This suggests using the screened Debye-Huckel potential,

$v_{i j}=\frac{z_{i} z_{i}}{r} e^{2} \exp \left(-r / \lambda_{d}\right)$, 
where the Debye screening length is (see DeWitt 1961):

$\lambda_{d}=\left(\frac{k T}{4 \pi e^{2} \Sigma_{\alpha} n_{\alpha} z_{\alpha}^{2} \Theta_{\alpha}}\right)^{1 / 2}$.

The sum in the denominator of the Debye length is over all charged particles in the plasma, including electrons. As the electrons become degenerate, they contribute less to polarization, and $\theta_{e}<1$ (see Fontaine et al. 1977).

The above integrals cannot be solved analytically when the Debye-Huckel potential is used. At sufficiently low density the collision integral is dominated by collisions with impact parameters less than the screening length, so the potential can be approximated as a pure Coulomb potential with the integration truncated at the Debye screening length. To find an analytic solution one must also replace $\mathrm{g}^{2}$ in the integrals for $\mathrm{X}_{\mathrm{ij}}$ with an assumed average value $\left\langle\mathrm{g}^{2}\right\rangle$ (typically a value of 2 or 3 is chosen). Analytically, it is then found that:

$\Omega_{i j}^{(11)}=\pi\left(\frac{z_{i} z_{j} e^{2}}{2 k T}\right)^{2}\left[\frac{\left(m_{i}+m_{j}\right) k T}{2 m_{i} m_{j}}\right]^{1 / 2} \ln \Lambda_{i j^{\prime}}$

where

$\ln \Lambda_{i j}=\ln \left(\right.$ constant $\left.\frac{2 k T \lambda_{d}}{z_{i} z_{j} e^{2}}\right)$.

This quantity, $\ln \Lambda_{i j}$, is often referred to as the Coulomb logarithm. The constant inside the logarithm depends on the approximations made and is similar to the value chosen for $\left\langle\mathrm{g}^{2}\right\rangle$ in (5). Because the constant appears under the logarithm, the argument is usually made that, when $\ln \Lambda_{i j}$ is sufficiently large, uncertainties in the constant of a factor of a few are unimportant. When similar approximations are made for the other collision integrals it is found that, $z_{i j}=0.6, z_{i j}=1.3$, and $z^{\prime \prime} i j$ $=2$.

The above approximations are unnecessary and can lead to serious errors in the collision integrals for the conditions appropriate for stellar interiors (Paquette et al. 1986). Without making any of these approximations, the collision integral $\Omega_{i j}^{(11)}$ can be written in the form above, but with a function $C_{\mathrm{ij}}$ in place of $\ln \Lambda_{\mathrm{ij}}$, that still depends only on the single parameter $2 \mathrm{kTN} / \mathrm{Z}_{\mathrm{j}} \mathrm{e}^{2}$. This function can be evaluated numerically for the whole range needed. It is found that in the low density limit $\ln \Lambda_{i j}$ is an excellent approximation if the constant in (9) is chosen to be about $2 / 5$.

In the interior of main sequence stars, the bulk of the ions are fully ionized hydrogen and helium, and heavier ions can be neglected when calculating the Debye screening length. This gives

$\ln \Lambda_{i j}=-19.26-\ln z_{i} z_{j}-\frac{1}{2} \ln \rho-\frac{1}{2} \ln \left[1+\left(\frac{x+1}{2}\right) \Theta_{e}\right]+\frac{3}{2} \ln T$,

when $\rho$ is in $\mathrm{gm} \mathrm{cm}^{-3}$ and $\mathrm{T}$ in $\mathrm{K}$. Specializing to collisions between $\mathrm{H}$ and $\mathrm{He}$ and taking $\theta_{e}=1$ gives, 


$$
\ln \Lambda_{X Y}=-19.95-\frac{1}{2} \ln \rho-\frac{1}{2} \ln \left(\frac{x+3}{2}\right)+\frac{3}{2} \ln T .
$$

For conditions characteristic of the radiative interior of the $\operatorname{Sun} \ln \Lambda_{x y} \simeq 2$, while in the centres of globular cluster turn-off stars it can be as small as 1.5 . As can be seen from figure 1 , using $\ln \Lambda_{x y}$ as an approximation for $C_{x y}$ gives answers within about $10 \%$ when $\ln \Lambda_{x y}>1$, but begins to diverge rather rapidly for $\ln \Lambda_{x y}<1$.

For heavy elements diffusing in stellar interiors, $\Lambda_{i j}$ is significantly smaller because of the $-\ln Z_{i} Z_{j}$ term in equation (10). For an element with $Z_{i}=8, \ln \Lambda_{i j} \sim$ 0 for collisions with $\mathrm{He}$ nuclei in the solar interior. The collisional effects for heavy elements will therufore be substantially smaller than might be naively expected from the $\left(Z_{i} Z_{j}\right)^{2}$ factor in equation (8). While $\ln \Lambda$ is clearly meaningless as an estimate of the integral $C_{i j}$ in this case, the numerical values of Paquette et al. can be used. Figure 2 shows values for $C_{i j}$ calculated using Paquette et al.'s fits for a model of the sun and for a model of a $0.75 \mathrm{M}_{\odot}$ star near core $\mathrm{H}$ exhaustion. Note that the values for collisions involving fully ionized $\mathrm{O}$ are much smaller than for collisions between $\mathrm{H}$ and $\mathrm{He}$. If a simple formula is desired instead, the fitting formula

$$
c_{i j}=\frac{1}{1.2} \ln \left[e^{\left(1.2 \ln \Lambda_{i j}\right)}+1\right]
$$

agrees with the numerically determined value for $C_{i j}$ to within $15 \%$ for $\ln \Lambda_{i j}>-4$.

The ratios of collision integrals $\left(z_{i j}, z_{i j}^{\prime}\right.$, and $\left.z^{\prime \prime}{ }_{i j}\right)$ also differ substantially from the values they assume in the low density limit (see figure 3). Near $\ln \Lambda \mathrm{ij}=2, \mathrm{z}_{\mathrm{ij}}$ is $30 \%$ smaller than in the low density limit. As a result, for small $\ln \Lambda \mathrm{ij}$, thermal diffusion effects are smaller than given by estimates using the ratios appropriate for low densities.

For a given definition of the collision integrals there are well defined values for the resistance/diffusion coefficients. The arbitrary constant that is often found inside the Coulomb logarithm is a result of approximations that are only necessary if a simple analytic form for the integral is desired. It is wrong to take the range of formulae for the Coulomb logarithm that have appeared in the physics literature as giving the intrinsic theoretical uncertainty for diffusion in stellar interiors. (Especially as many of these formulae were derived by assuming that factors of 2 or so under the logarithm are unimportant). Numerical solutions to the collision integrals for a screened Coulomb potential have been calculated by Paquette et al. which avoid this uncertainty, and give the proper value in the low density limit where a simple analytic formula is meaningful.

This is not to say that there are no uncertainties in the diffusion velocities calculated with these collision integrals. The real uncertainties come from the physical assumptions involved in the initial definitions of the collision integrals and diffusion equations, not from the mathematical simplifications needed to derive simple analytic expressions. For instance, Roussel-Dupré (1982) estimates that including higher order moments of the velocity distribution function can change diffusion coefficients for H-He mixtures by about 10\%. See Michaud (1991) for a discussion of other potential uncertainties.

The assumption that most of the collisions are weak (i.e. the Fokker-Planck approximation) is equivalent to the requirement that the kinetic energy of the ions is large compared to their interaction energy. This is true when $\Lambda_{\mathrm{ij}}>>1$, but becomes increasingly less valid as $\Lambda_{i j}$ decreases. The collision integrals discussed above should therefore be reliable for $\mathrm{H}-\mathrm{He}$ plasmas in the interior of main-sequence stars, while the results for heavy ions in the same $\mathrm{H}-\mathrm{He}$ background are extrapolated 


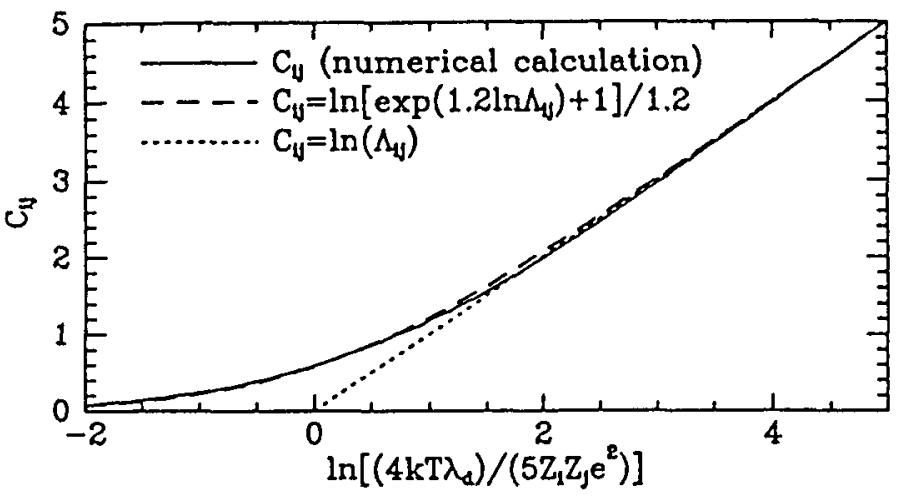

Fig. 1: Comparison of different approximations for $C_{\mathbf{i}}$.

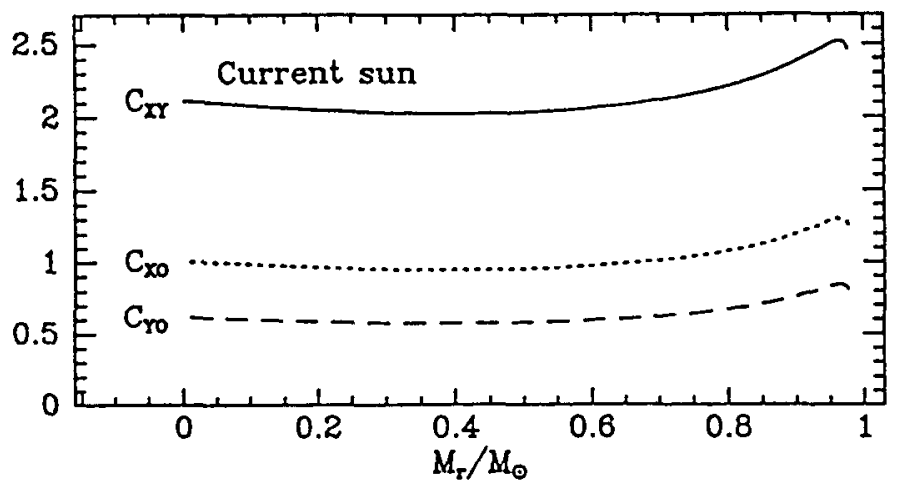

Fig. 2a: $C_{i j}$ for $\mathrm{H}, \mathrm{He}$, and $\mathrm{O}$ in a model of the current sun.

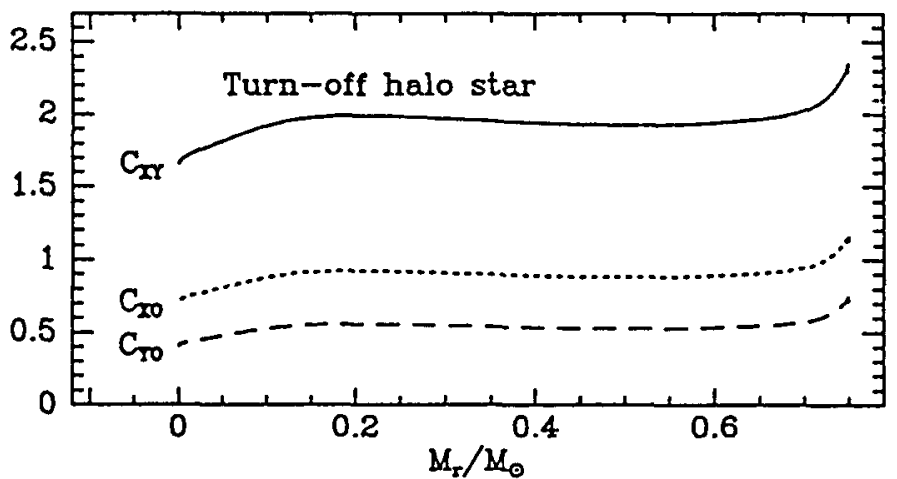

Fig. 2b: As in fig. 2a, but for a model of a $0.75 M_{\odot}$ star. 
beyond the regime where they are rigorously valid. Paquette et al. have shown that their results are in good agreement with calculations done in the limit of a very strongly coupled plasma. This suggests that the extrapolation may be valid for the intermediate coupling region. While this approach provides the best currently available estimates, it is very difficult to give quantitative estimates of accuracy.

\section{Simplified Formula for Diffusion Velocities.}

Given a value for the average velocity $V_{i}$ with respect to the centre of mass of a given ionic species at each point in the stellar interior, the rate of change of the mass fraction abundance $X_{i}$ of that species is given by

$\frac{d x_{i}}{d t}=-\frac{1}{\rho r^{2}} \frac{d}{d r}\left(\rho r^{2} v_{i} x_{i}\right)$

It is often useful to have available simple formulae for $V_{i}$ for use in this equation. The formulae given below are for a mixture of fully ionized $\mathrm{H}$ and ${ }^{4} \mathrm{He}$ with other elements present only in trace amounts. Such formulae must be compared with the solutions of the full system of equations (1) to check their range of validity. Numerical results will be compared to a variety of simpler formulations.

\subsection{A simplified formula for fully ionized $\mathrm{H}$-He mixtures.}

If the heat flux terms in equation (1) are ignored (i.e. $z_{i j}=0$ ) and if it is assumed that $C_{i j}$ can be approximated by $\ln \Lambda_{i j}$, then the diffusion velocity for $\mathrm{H}$ in a $\mathrm{H}-\mathrm{He}$ mixture becomes,

$V_{H}=-\frac{B T^{5 / 2}}{\rho \ln \Lambda_{i j}}\left[\frac{5}{4}(1-X) \frac{d \ln P}{d r}+\frac{3+X}{(1+X)(3+5 X)} \frac{d \ln x}{d r}\right]$

where, to simplify writing this expression, the constant

$B=\frac{15}{16}\left(\frac{2 m_{0}}{5 \pi}\right) 1 / 2 \frac{k^{5 / 2}}{e^{4}}$

has been defined. This formula is identical to that implied by equation (41) of Bahcall and Loeb (1990) (B\&L). However, for a trace $\mathrm{H}$ abundance, equation (14) underestimates the diffusion velocity obtained with a complete solution by about $30 \%$. Examination of equation (18.2) of Burgers (1969) shows that the heat flux terms, the $r_{i}$ 's, are non-zero even in the absence of a temperature gradient. (The equivalent corrections are called the second approximation in Chapman and Cowling). An excellent approximation to the results of equation (1) can be obtained if we multiply formula (14) for $V_{H}$ by the factor $1 /(0.7+0.3 X)$ (see figure 4 where $\mathrm{V}_{\mathrm{H}}-\mathrm{V}_{\mathrm{He}}=\mathrm{V}_{\mathrm{H}} /(1-\mathrm{X})$ is plotted).

When a non-zero temperature gradient is included, the rate of settling increases due to thermal diffusion. By comparing velocities calculated from the full system of equations with and without a temperature gradient, it is found that the fraction of the total diffusion velocity for $\mathrm{H}$ and $\mathrm{He}$ due to thermal diffusion,

$R_{t h} \equiv \frac{v(\nabla \neq 0)-v(\nabla=0)}{v(\nabla=0)}=0.9 \nabla$

in the interiors of main sequence stars. Typically, this implies that thermal diffusion 
increases the total diffusion velocity by about $30 \%$. The value of $R_{t h}$ varies only slightly with the $\mathrm{H}-\mathrm{He}$ ratio and $\ln \Lambda_{x y}$, and the formula

$$
\begin{aligned}
V_{H}=-\frac{B T^{5 / 2}}{\rho \ln \Lambda_{i j}(0.7+0.3 X)}\left(\left(\frac{5}{4}+1.125 \nabla\right)\right. & (1-X) \frac{d \ln P}{d r} \\
& \left.+\frac{(3+X)}{(1+X)(3+5 X)} \frac{d \ln X}{d r}\right]
\end{aligned}
$$

reproduces the total diffusion velocity as calculated for the full system to within $10 \%$ for $\ln \Lambda_{x y}>2$. For smaller values of $\ln \Lambda_{x y}$ the accuracy begins to degrade. By using the approximation given by equation (12) in place of $\ln \Lambda_{x y}$ the usefulness of the above formula can be extended, and answers within $20 \%$ can be obtained for $\ln \Lambda_{x y}$ near 1. Figure 4 shows $\mathrm{V}_{\mathrm{H}}-\mathrm{V}_{\mathrm{He}}$ calculated with $B \& L$ 's assumptions together with our formula for $\ln \Lambda_{x y}$ (equation 11). Most of the difference between B\&L's result and equation (17) is due to the extra heat-flux terms discussed above. If the formula for $\ln \Lambda_{L}$ given by Chaboyer et al.(1992) is used, smaller diffusion velocities are found. However there are numerous reasons for preferring our value for $\ln \Lambda_{\mathrm{ij}}$.

\subsection{Simple formula for a trace element in a H-He background}

Ignoring the heat flux terms in equation (1), the velocity of a trace element $i$ in a fully ionized $\mathrm{H}-\mathrm{He}$ mixture can be written as,

$$
\begin{aligned}
& v_{i}=-\frac{2 B T^{5 / 2}}{5^{1 / 2}{ }_{\rho z_{i}^{2}}^{2}}\left[\frac{\frac{d}{d r}\left\{\ln \left[\frac{x_{i}}{5 X+3}\left(\frac{1+X}{5 X+3}\right]_{i}\right]\right\}+\left[1+z_{i}-A_{i} \frac{5 X+3}{4}\right) \frac{d \ln P}{d r}}{x\left(A_{i x}^{1 / 2} c_{i x}-A_{i y}^{1 / 2} c_{i y}\right)+A_{i Y}^{1 / 2} c_{i y}}\right] \\
& +X v_{H} \frac{\left(A_{i x}^{1 / 2} c_{i x}-A_{i y}^{1 / 2} c_{i y}\right)}{x\left(A_{i x}^{1 / 2} c_{i x}-A_{i Y}^{1 / 2} c_{i y}\right)+A_{i Y}^{1 / 2} c_{i y}} \text {. }
\end{aligned}
$$

Here $X_{i}$ is the mass fraction of the trace element, and $X$, without a subscript, is the mass fraction of hydrogen while $A_{i x}$ is the reduced mass (in atomic number units) of the trace element with hydrogen. If it is assumed that $C_{i x}=C_{i y}=\ln \Lambda_{x y}$ and $A_{i}>>A_{y}$, then equation (53) of B\&L can be recovered (apart from an extra erroneous factor of $X$ that B\&L included in the composition gradient term). However, these approximations should not be made, as they lead to qualitatively different results for the settling of heavy elements. As can be seen from the discussion in section 2 , the factor $C_{i j}$ depends strongly on the product $Z_{i} Z_{j}$, especially when $\ln \Lambda_{i j}$ is small.

The first term in equation (18) can be identified as the settling velocity of the trace element in a fixed background, while the second term represents the net drag on the trace element from collisions with moving $\mathrm{H}$ and $\mathrm{He}$ ions. The approximation that B\&L made results in a substantial overestimate of the strength of the collisions of the heavy elements with $\mathrm{H}$ and $\mathrm{He}$. For typical parameters the settling term is underestimated by a factor of 2 to 3 , and the drag term is overestimated by 3 to $5 \mathrm{x}$. The drag term then dominates the total diffusion velocity. However, if formula (18) is used with either the numerically calculated values for $\mathrm{C}_{\mathrm{ij}}$ or the approximation given by equation (12), then the drag term makes only a minor contribution to the total diffusion velocity.

While the results from formula (18) agree quite well with the solution of the 


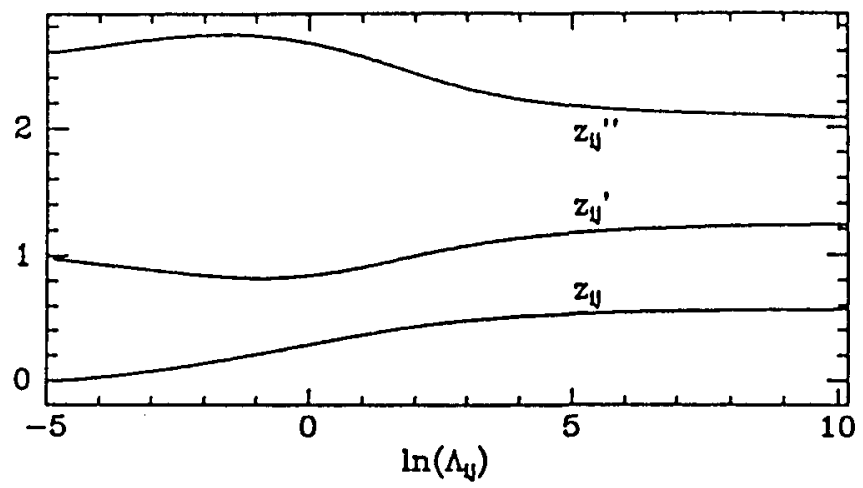

Fig. 3: The heat flux coefficients as a function of $\ln \Lambda_{i j}$.

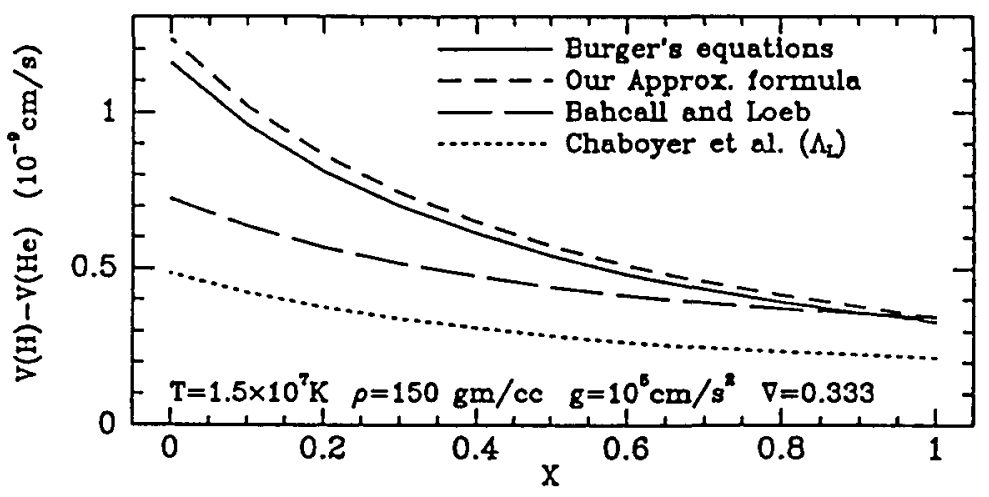

Fig. 4: Relative diffusion velocities of $\mathrm{H}$ and $\mathrm{He}$ as a function of $X$.

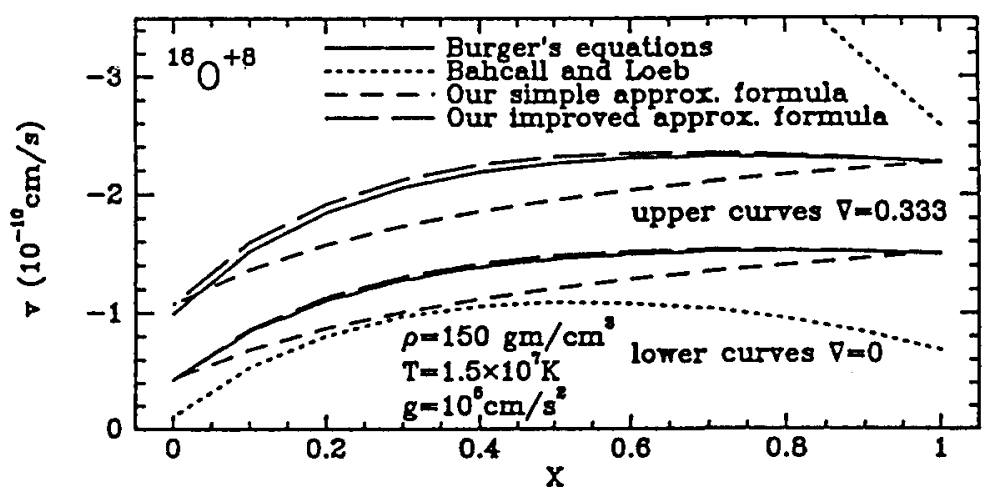

Fig. 5: Diffusion velocity for ${ }^{16} \mathrm{O}^{+8}$ in a H-He mixture. 
full system of equations (1) for pure $\mathrm{H}$ or pure $\mathrm{He}$, it significantly underestimates the velocity for intermediate values. Comparison to the full system of Burgers' equations reveals that the discrepancy comes from ignoring the heat flux terms in deriving equation (18). If equation (1) is solved while artificially setting $z_{\mathrm{ij}}$ to 0 , then the results agree well with the values calculated by formula (18). This extra drag can be reasonably approximated by adding $\sim-.23 \mathrm{XV}_{\mathrm{x}}$ to the velocity given above.

For conditions characteristic for the interiors of main sequence stars, we find that the thermal diffusion velocity can be approximated (within $20 \%$ ) by adding the empirically determined quantity,

$v_{\text {therm }}=\frac{0.54 B(4.75 X+2.25) \mathrm{T}^{5 / 2}}{\rho\left(\ln \Lambda_{x y}+5\right)} \nabla \frac{\mathrm{d} \ln \mathrm{p}}{\mathrm{dr}}$

to the total diffusion velocity for heavy ions.

Figure 5 shows the $\mathrm{V}$ for ${ }^{16} \mathrm{O}$ as a function of $\mathrm{X}$ computed in a variety of ways. The improved formula curve includes the correction term $-.23 \mathrm{XV}$ x discussed above. Even in the absence of a temperature gradient, the approximations of $B \& L$ underestimate diffusion velocities by a factor of up to three, especially in pure $\mathrm{H}$ and pure He. The formula given by B\&L for the thermal diffusion velocity of heavy elements is proportional to $1 / \mathrm{X}$.

\section{III- EVOLUTIONARY RESULTS IN POPULATION I STARS.}

\section{The Sun}

A number of authors have estimated gravitational settling in the Sun (Aller and Chapman 1960, Noerdlinger 1977, Wambsganss 1988, Cox et al. 1989, Proffitt \& Michaud 1991b, Bahcall \& Pinsonneault 1992). Disagreements have been analyzed by Proffitt and Michaud (1991b) and it appears that, if diffusion is not inhibited by mixing below the convection zone, the current surface mass fraction helium abundance of the Sun, $Y_{\text {, }}$, should be about 0.03 (10\%) lower than the initial solar helium abundance. Models with diffusion have slightly higher central densities $(0.5 \%)$ and temperatures (2\%). A slightly larger mixing length is also needed. Predicted neutrino fluxes are slightly increased.

The assumption that there is no mixing below the base of the surface convection zone is probably incorrect, as the current solar $\mathrm{Li}$ abundance is 100-200 times smaller than the initial value and it appears difficult to account for all of the depletion by pre-main sequence burning. While the cause and nature of main sequence mixing is controversial, observational data can constrain its magnitude. Figure 2 of Proffitt and Michaud (1991b) shows how two possible distributions of turbulent mixing that approximately reproduce the observed $\mathrm{Li}$ depletion affect the surface settling of $\mathrm{He}$. It does not seem possible to reduce the surface settling by more than a factor of two without over-depleting $\mathrm{Li}$, but the shape of the interior $\mathrm{He}$ profile and the steepness of the composition gradient can be quite sensitive to the details.

Observations of solar p-modes provide a means for directly measuring the $\mathrm{He}$ abundance of the surface convection zone and the sound speed as a function of radius (see the discussion in Dziembowski et al. 1992). These measurements are potentially sensitive enough to distinguish between the different profiles shown in Figure 2 of Proffitt and Michaud (1991b) and suggest a surface He abundance consistent with the diffusion predictions noted above (see also Guzik \& Cox 1992). Once current 
uncertainties in other physics (opacities, abundances, and convection theory) are resolved, the oscillations should eventually provide a direct test of turbulent mixing in $\mathrm{G}$ dwarfs.

\section{The Li Gap}

The observation by Boesgaard ant Tripicco (1986) of a well defined gap in the $\mathrm{Li}$ abundance curve as a function $\mathrm{T}_{\text {ef }}$ in the Hyades (see their Fig. 2; see also Fig. 2.1 of Michaud and Charbonneau 1991) is suggestive of a competition between gravity and $g_{R}(\mathrm{Li})$ as the convection zone becomes deeper with decreasing $T_{\text {eff }}$ (Michaud 1986).

The $\mathrm{T}_{\text {erf }}$ interval covered by the $\mathrm{Li}$ gap stars in the Hyades is within a few hundred degrees of the region where the mass in the convection zone increases rapidly. The agreement between the observed and calculated $T_{\text {efr }}$ of the deepest part of the gap depends on the value of $\alpha$ used for the models as well as on the opacity tables. Helium settling occurs at the same time as Li settling since the two diffusion velocities are nearly equal (Michaud 1986). To make precise comparisons between the observed Li gap in clusters and abundances to be expected from standard stellar evolution, it is necessary to take into account the settling of He (Richer et al. 1992).

In stars of $1.0<\mathrm{M}<1.5 \mathrm{M}_{\odot}$, the evolutionary time scale varies from $10^{10}$ to $10^{9} \mathrm{yr}$ while the diffusion time scale within the core is about $10^{11} \mathrm{yr}$. While, in the Sun, gravitational settling has increased the He abundance in the He burning region by $5 \%$ of the original value (Cox, Guzik and Kidman 1989, Noerdlinger 1977, Proffitt and Michaud 1991), the increase is negligible in $1.5 \mathrm{M}_{\odot}$ stars during the main sequence. The effects of evolution come from abundance changes in the centre, while diffusion causes abundance changes mainly in the envelope for stars of $\mathrm{M}>1.4 \mathrm{M}_{\odot}$. This allowed Richer $e t$ al. to calculate the effects of diffusion only in the envelope and use the evolutionary calculations without diffusion for the centre of such stars.

Using various opacity tables and detailed calculations of the radiative acceleration of $\mathrm{Li}$, Richer and Michaud (1992) also calculated the $\mathrm{Li}$ gap as a function of cluster age. They calibrated the value of $\alpha$ using the Sun (see $\$$ III-1).

It is then found that: a) the position of the $\mathrm{Li}$ gap is nearly exactly the same for all opacity tables; b) the position of the $\mathrm{Li}$ gap is at $\mathrm{T}_{\text {eff }}=6740 \mathrm{~K}$, in agreement with observations; c) the depth of the $\mathrm{Li}$ gap varies with opacity tables. It is by a factor of 100 at the age of the Hyades using the OPAL tables but is only by a factor of 10 using the Los Alamos tables; d) the width of the gap is too narrow (about 150 instead of $250 \mathrm{~K}$ ). However the very abrupt blue side of the gap is explained; e) as clusters become older the position of the gap shifts to slightly lower $T_{\text {eff }} ; f$ for stars with $\mathrm{T}_{\mathrm{eff}}>7000 \mathrm{~K}$, there is a need for an alternate hydrodynamical process competing with diffusion. This is the AmFm domain where there is a need for such a process to reduce the anomalies that uninhibited diffusion leads to.

\section{A and B type stars}

The evolutionary time scale for B and A stars vary from $10^{8}$ to $10^{9}$ yr while the diffusion time scale in the centre is around $10^{11} \mathrm{yr}$ so that the effects of diffusion in the centre are small enough to be neglected. The effects are much larger in the external regions. Since the convection zone is of order $10^{-10} \mathrm{M}_{\odot}$ and the helium settling time scale, $\theta$, varies approximately as (Michaud 1977) $2.310^{11} \Delta \mathrm{M}^{0.545} \mathrm{yr}$, where $\Delta \mathrm{M}$ is the mass in the convection zone in $\mathrm{M}_{\odot}$, the time scale for the disappearance of the He convection zone is of order of $10^{6} \mathrm{yr}$, much shorter than the evolutionary time scale. The diffusion time scales become even shorter once the He 
convection zone has disappeared. Since the largest observed abundance anomalies are observed in those stars, Michaud (1970) suggested that radiation driven diffusion was responsible for abundance anomalies observed in the ApBp stars.

The simplest of these stars to model are the AmFm stars. They are cool enough to have a $\mathrm{H}$ convection zone and they have no large organized magnetic field so that chemical separation occurs only below the convection zone. Immediately below the hydrogen convection zone, $\mathrm{Ca}$ and $\mathrm{Sc}$ are in a rare gas configuration so that the radiative acceleration on them is smaller than gravity and they sink as is observed (Watson 1971; Smith 1973; Cayrel Burkhart and Van't Veer 1991). This property is naturally explained by the parameter free evolutionary calculations, since these lead to the disappearance of the $\mathrm{He}$ convection zone because of gravitational settling. This also explains the absence of $\delta$ Scuti pulsators among the classical Am stars (Baglin 1972).

One may now also test the parameter free evolutionary calculations by comparing the $T_{\text {eff }}$ where the AmFm phenomenon occurs with theoretical caiculations. Then the effect of He settling on the $T_{\text {eft }}$ and on the size of the convection zone (Vauclair, Vauclair and Pamjatnikh 1974), as well as evolutionary effects have to be taken into account (Richer et al. 1992). The red edge of AmFm phenomenon should be determined theoretically within at most $100 \mathrm{~K}$. Calculations are in progress (Richer and Michaud 1992a).

At the same time as the $\mathrm{Ca}$ and $\mathrm{Sc}$ underabundances are explained, overabundances of most elements heavier than $\mathrm{Mg}$ are expected and observed (see Michaud et al. 1976). The model correctly predicts which elements are overabundant and which are underabundant, with few exceptions. The expected anomalies are however larger than observed unless a competing hydrodynamical process is present. Turbulence cannot be the only process, since it wipes out the Sc and $\mathrm{Ca}$ anomalies before reducing the overabundances (Vauclair Vauclair and Michaud 1978). Mass loss, of the order of $10^{-15} \mathrm{M}_{\odot} \mathrm{yr}^{-1}$, appears to be compatible with both (Michaud et al. 1983) though it remains to be seen if it can be compatible with the Li observations in clusters (Richer et al. 1992).

\section{IV- DIFFUSION IN POP. II DWARFS}

Pop. II stars are older than the Sun, and many have thinner surface convection zones, so that the effects of diffusion are larger than in Pop. I stars (Noerdlinger and Arigo 1980, Stringfellow et al. 1983). The central settling of He causes core $\mathrm{H}$ exhaustion to occur earlier and at a lower luminosity, while surface settling causes an increase in the stellar radius and a decrease in the $T_{\text {eff }}$ relative to non-diffusion models. The total shift in the turnoff $T_{\text {eff }}$ between diffusion and non-diffusion models is typically about $200 \mathrm{~K}$. Stringfellow et al. concluded that this resulted in a $14-25 \%$ decrease in the ages of globular clusters. Proffitt and VandenBerg (1991) using Paquette et al.'s diffusion rates concluded that $\mathrm{H}-\mathrm{He}$ diffusion results in a $10 \%$ decrease in the ages of globular clusters as measured by the luminosity of the turn-off (see also Chaboyer et al. 1992). The ${ }^{7} \mathrm{Li}$ is expected to settle at about the same speed as ${ }^{~} \mathrm{He}$, and can be used to constrain surface settling and mixing. Spite and Spite (1982) discovered that metal poor halo stars with $[\mathrm{Fe} / \mathrm{H}]<-1.3$ and Teff $>5500 \mathrm{~K}$ all have essentially the same $\mathrm{Li}$ abundance, with very little star to star scatter, and no obvious trend with $\mathrm{T}_{\text {eff }}$ or metallicity (Deliyannis et al. suggest an approximately $10 \%$ intrinsic scatter). However, models that include gravitational settling without any extra-mixing predict a noticeable decrease in the surface $\mathrm{Li}$ abundance towards the hot end of the plateau, due to gravitational settling of $\mathrm{He}$ and $\mathrm{Li}$ from the thin convection zones of these stars (see Proffitt and Michaud 1991a). To some extent 
this decrease towards high $T_{\text {eff }}$ can be cancelled by increased pre-main-sequence depletion at cooler $T_{\text {eff }}$ but the models still seem to predict an unobserved Pop. II analogue of the Pop. I F-star Li gap (but see G186-26 with Li down by at least 0.9 dex, Hobbs et al. 1991). Either (1) uncertainties, perhaps due to reddening, in the $T_{\text {eff }}$ of the stars at the hot end of the halo sequence erroneously extend it, (2) the Paquette et al. diffusion calculations significantly overestimate atomic diffusion, (3) these models of Proffitt and Michaud significantly underestimate the thickness of the surface convection zone for the warmest halo dwarfs, or (4) some kind of mixing below the surface convection zone moderates the amount of gravitational settling.

We have already discussed why we believe that the Paquette et al. calculations are currently the best available.

The thickness of the surface convection zones is, however, uncertain for both theoretical and observational reasons. The expected mass of the surface convection zones in very metal poor dwarfs changes from about $10^{-2} \mathrm{M}_{\odot}$ near $6000 \mathrm{~K}$ to $<10^{-4}$ $M_{\odot}$ at $6400 \mathrm{~K}$, but the exact values are very sensitive to uncertain details of convection theory, opacities, assumed mixing length, and colour $-T_{\text {eff }}$ conversions, including potential reddening corrections.

Calculating detailed interaction between gravitational settling and turbulence below the convection zone requires a model of turbulence. Pinsonneault, $e t$ al. (1992) have proposed a model for turbulent mixing in halo stars but they have not yet simultaneously considered the interaction between this turbulence and gravitational settling. Some constraints can be studied by simply enforcing differing minimum masses for the fully mixed surface region. We have constructed a number of models of $0.75 \mathrm{M}_{\odot}$ halo stars, in which we have set various minimum masses for the fully mixel surface region (i.e. we assumed a very large turbulent diffusion coefficient in the outer $\mathrm{n} \%$ of the mass but allowed diffusion to be uninhibited below this). Eliminating a noticeable Li dip at the hot end of the Spite plateau requires keeping the outer $1-2 \%$ of the stellar mass well mixed, while forcing the outer $5 \%$ of the mass to always be well mixed will destroy $90 \%$ of the $\mathrm{Li}$ via nuclear burning. However, regardless of how the depth of the mixed surface zone is chosen, if the Paquette et al. diffusion rates are used, the combination of gravitational settling and nuclear burning ensures that the surface $\mathrm{Li}$ abundance at $15 \mathrm{Gyr}$ will be AT MOST $70 \%$ of the ZAMS value. We do not believe that any distribution of turbulent mixing or overshooting would allow a higher $\mathrm{Li}$ abundance at this age. The reduction in surface settling caused by this assumed extra-mixing does not completely eliminate the larger radii and shift towards smaller $T_{\text {eff }}$ that the He settling causes. Even when the outer $5 \%$ of the mass is thoroughly mixed, the difference in turn-off $\mathrm{T}_{\text {eff }}$ between diffusion and non-diffusion models is only reduced by half.

\section{REFERENCES}

Alecian, G., and Artru, M. -C., 1990, A\&A, 234, 323. Alecian, G., Michaud, G., and Tully, J. 1992, preprint. Aller, L. H., and Chapman, S. 1960, ApJ, 132, 461.

Baglin, A. 1972, A\&A, 19, 45.

Bahcall, J. N., and Loeb, A. 1990, ApJ, 360, 267.

Bahcall J. N., \& Pinsonneault, M. H. 1991, ApJ, submitted

Boesgaard, A. M., and Tripicco, M. J. 1986, ApJL, 302, L49.

Burgers, J. M. 1969, Flow Equations for Composite Gases, (New York: Academic Press).

Cayrel, R., Burkhart, C., and Van't Veer, C. 1991, in IAU Symposium 145, Evolution of Stars: the Photospheric Abundance Connection, Golden Sands, 
Bulgaria, 25-31 august, ed. G. Michaud and A. Tutukov (Kluwer), pp. 99-110. Chaboyer, B., Deliyannis, C. P., Demarque, P., Pinsonneault, M. H., \& Sarajedini, A. 1992, ApJ, 388, 372

Chapman, S., and Cowling, T. G. 1970, The Mathematical Theory of non-uniform Gases (3d ed.; Cambridge: Cambridge University Press).

Charbonneau, P., and Michaud, G. 1988, ApJ, 327, 809.

Charbonneau, P., and Michaud, G. 1991, ApJ, 370, 693.

Cox, A. N., Guzik, J. A., and Kidman, R. B. 1989, ApJ, 342, 1187.

Deliyannis, C. P., Pinsonneault, M. H. \& Duncan, D. K. 1992, ApJ, submitted

Deliyannis, C. P., Pinsonneault, M. H. \& Demarque, P. 1992, ApJS, 78,

DeWitt, H. E. 1961, J. Nucl. Energy C, Plasma Physics, 2, 27.

Dziembowski, W. A., Pamyatnykh, A. A., and Sienkeiwicz, R. 1992, preprint

Eddington, A. S. 1926, The Internal Constitution of the Stars (New York: Dover [1959] reprint), § 199.

Fontaine et al 1977.

Guzik, J. A., \& Cox, A. N. 1992, ApJL, 386, 729.

Hobbs, L. M., Welty, D. E. \& Thornburn, J. A. 1991, ApJ 373, L47

Kurucz, R. L. 1991, in Stellar Atmospheres: Beyond Classical Models, ed. by L. Crivellari, I. Hubeny and D. G. Hummer, NATO ASI Series (Dordrecht, Kluwer), p. 440 .

Michaud, G. 1970, ApJ, 160, 641.

Michaud, G. 1977, Nature, 266, 433.

Michaud, G. 1982, ApJ, 258, 349.

Michaud, G. 1986, ApJ, 302, 650.

Michaud, G. 1987, Physica Scripta, 36, 112.

Michaud, G. 1991, Annales de Physique Fr., 16, 481.

Michaud, G., and Charbonneau, P. 1991, Space Science Reviews, 57, 1.

Michaud, G., Charland, Y., Vauclair, S., and Vauclair, G. 1976, ApJ, 210, 447.

Michaud, G., Tarasick, D., Charland, Y., and Pelletier, C. 1983, ApJ, 269, 239.

Noerdlinger, P. D. 1977, A\&A, 57, 407.

Noerdlinger, P. D. \& Arigo, R. J. 1980, ApJL, 237, L15

Paquette, C., Pelletier, C., Fontaine, G., and Michaud, G. 1986, ApJS, 61, 177.

Proffitt, C. R. \& Michaud, G. 1991a, ApJ, 371, 584

Proffitt, C. R. \& Michaud, G. 1991b, ApJ, 380, 238

Proffitt, C. R. \& VandenBerg, D. A. 1991, ApJS, 77, 473

Richer, J., and Michaud, G. 1992, in preparation.

Richer, J., Michaud, G., and Proffitt, C. 1992, ApJS, in press.

Rogers, F. J., and Iglesias, C. A. 1992, ApJS, 79, 507.

Roussel-Dupre, R. 1981, ApJ, 243, 329.

Roussel-Dupre, R. 1982, ApJ, 252, 393.

Smith, M. A. 1973, ApJS, 25, 277.

Spite, F. \& Spite, M. 1982, A\&A, 115, 357

Stringfellow, G. S., Bodenheimer, P., Noerdlinger, P. D. \& Arigo, R. J. 1983, ApJ, 264,228

Vauclair, G., Vauclair, S., and Michaud, G. 1978, ApJ, 223, 920.

Vauclair, G., Vauclair, S., and Pamjatnikh, A. 1974, A\&A, 31, 63.

Wambsganss, J. 1988, A\&A, 205, 125.

Watson, W. D. 1971, A\&A, 13, 263. 